\title{
An Interesting Case of Post Traumatic Tibialis Anterior Muscle Herniation Hegde AS
}

\author{
Department of Orthopaedics \\ Yenepoya Medical College \\ Deralakatte, Mangalore \\ India
}

Corresponding Author

Atmananda S.Hegde

Department of Orthopaedics

Yenepoya Medical College

Deralakatte, Mangalore

India

E-mail: atmanandahegde@gmail.com

Citation

Hegde AS. An Interesting Case of Post Traumatic Tibialis Anterior Muscle Herniation. Kathmandu Univ Med J 2014;44(4):332-334.

\begin{abstract}
A muscle hernia is defined as a protrusion of the muscle belly through an acquired or congenital fascial defect. Muscle herniation through fascia is a relatively rare entity. Though predominantly asymptomatic, rarely they can be cause of vague pain in the leg, aggravated by exercises. Various conservative measures have been described for asymptomatic hernias, but treatment of symptomatic cases remains controversial. Here we present a case of symptomatic post traumatic tibialis anterior muscle herniation which was treated successfully with autologous fascia lata graft in Yenepoya Medical College, Mangalore in the month of January 2013.

Muscle hernias should be kept in mind as a rare differential diagnosis whenever patients present with persisting vague leg pain with or without swelling. If conservative treatment fails, we recommend closure with autologous graft or fasciotomy to relieve the symptoms.
\end{abstract}

\section{KEY WORDS}

Fascia lata graft, hernia, tibialis anterior

\section{INTRODUCTION}

A muscle hernia is defined as a protrusion of the muscle belly through an acquired or congenital fascial defect. Muscle herniation through fascia is a relatively rare entity. It can be post traumatic or constitutional. Occupational and sporting activities like foot soldier's marching, mountain climbing, skiing and athletics have been implicated as causes. ${ }^{1}$ Large symptomatic hernias are rare. Though predominantly asymptomatic, rarely they can be cause of vague pain in the leg, aggravated by exercises. Clinical diagnosis is challenging and requires investigations like sonography and MRI. Various conservative measures have been described for asymptomatic hernias, but treatment of symptomatic cases remains controversial. Here we present a case of symptomatic post traumatic tibialis anterior muscle herniation which was treated successfully with autologous fascia lata graft.

\section{CASE-REPORT}

A 26 year old manual labourer presented to the Department of Orthopaedics, Yenepoya Medical College, Mangalore, in January 2013 with complaints of swelling in the anterior aspect of left leg associated with dull aching pain since last two years. There was a history of blunt trauma to the same area due to horse kick. Swelling used to increase in size during prolonged standing and exertion with increase in severity of pain.

On examination of left leg, there was a diffuse bulge in the anterolateral aspect of middle third of leg (Fig 1), with indistinct borders. It was mildly tender with no local rise of temperature. Surface of the swelling was smooth with soft to firm consistency. On deep palpation a defect could be felt. It was non pulsatile and partially compressible. It was not fixed to the overlying skin and became more prominent on actively dorsiflexing the ankle. There were no distal 


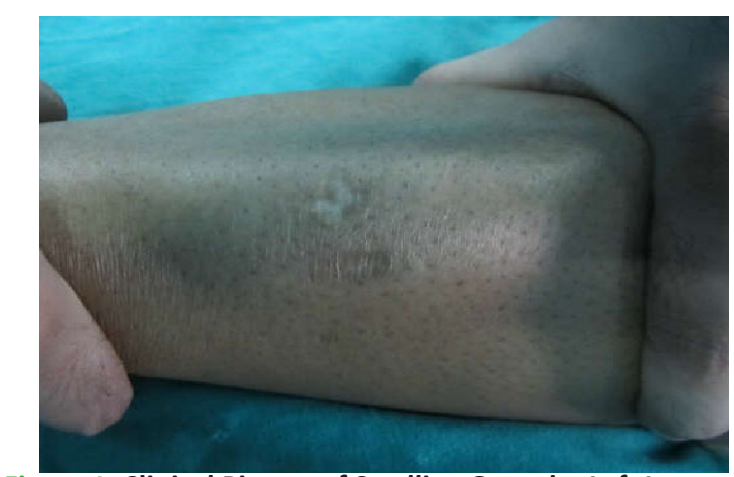

Figure 1. Clinical Picture of Swelling Over the Left Leg.

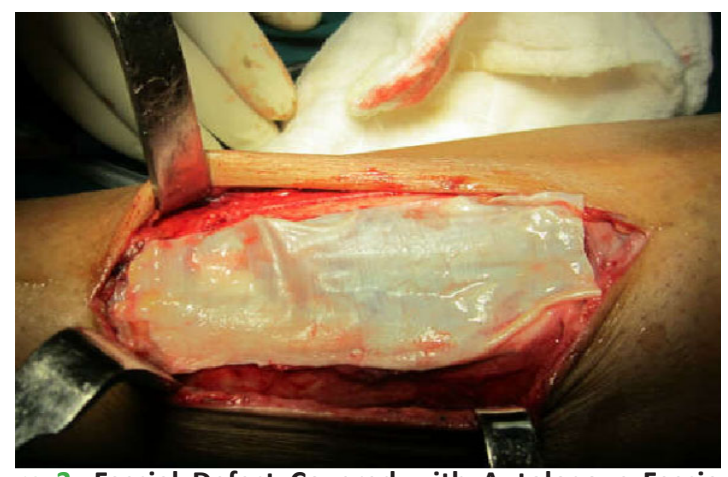

Figure 3. Fascial Defect Covered with Autologous Fascia Lata Graft.

neurovascular deficits.

Based on history and clinical findings, tibialis anterior herniation was suspected and it was confirmed by sonography. Conservative management in the form of compression bandage application did not give him relief. In view of disabling symptoms, surgical treatment was offered to him. A fully informed consent was taken prior to the surgery. During the surgery, longitudinal skin incision overlying the swelling was used and a defect in the deep fascia measuring $12 \mathrm{cms} \times 5 \mathrm{cms}$ in width was noted through which the tibialis anterior muscle was bulging out (Fig 2). Deep fascia was undermined and underlying muscle separated. Then fascia lata graft of the required dimension was taken from the ipsilateral thigh and was spread over the defect (Fig 3). Graft was sutured to the surrounding deep fascia sutured maintaining sufficient tension by interrupted mattress knots. Both the donor and host areas were closed layers after achieving haemostasis. Compression dressings were applied. Patient was allowed full weight bearing mobilisation after five days and sutures were removed after 10 days. Compression bandage was used for two months. At two months follow-up he was found to be symptom free and was advised to return to work.(Fig 4)

\section{DISCUSSION}

Muscle herniations can be found at various sites of the body, with the lower leg being the most common. Though rare, these may be underdiagnosed or misdiagnosed as hematomas and varicosities. ${ }^{2}$ About 200 cases of muscle

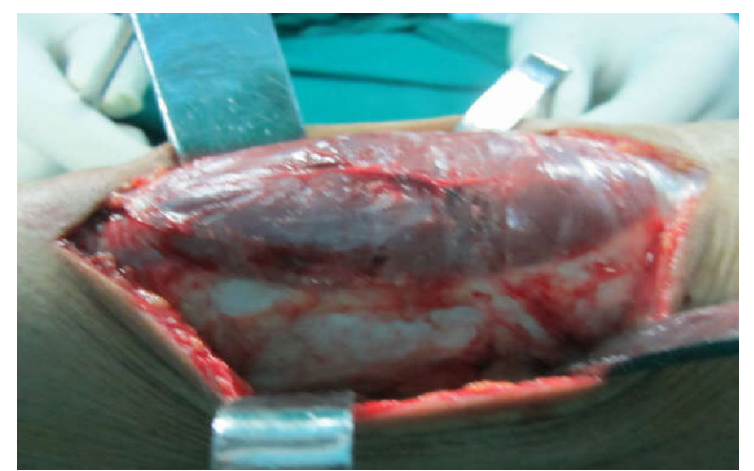

Figure 2. Intra-Operative Pictures Showing the Rent in the Fascia with the Underlying Muscle Bulging Out.

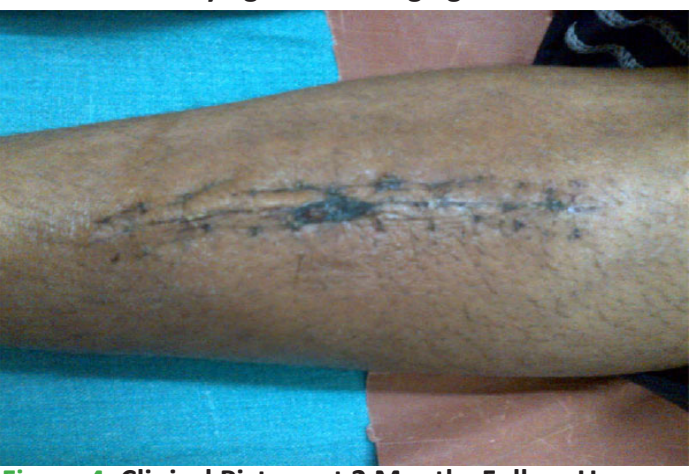

Figure 4. Clinical Picture at 2 Months Follow-Up.

hernias have been described in literature. Most of the cases are small in size and described in athletes and young adults requiring excessive strain on legs. ${ }^{3,4}$ Because of superficial and tight fascial compartment, anterolateral tibial compartment is the commonest site affected by muscle herniation. ${ }^{3-5}$

Muscle herniation can be classified as traumatic or constitutional. Traumatic muscle hernias are either direct following an open injury to the leg, or indirect following a repeated blunt trauma to a contracted muscle causing rupture of the fascia and consequent herniation of the muscle. ${ }^{6}$ Constitutional hernias are caused by chronic stress on the fascia from the underlying muscle. It has been suggested that the fenestrations in the fascia through which perforating veins enter enlarge and eventually muscle hernias bulge through these openings in the fascia. ${ }^{7}$ In the case presented, blunt trauma to left leg due to horse kick two years back might have contributed to weakness of fascia overlying tibialis anterior muscle which led to symptomatic hernia later on. It is easier to diagnose small muscle hernias by local examination. When hernia is large, the diagnosis is difficult due to diffuse bulge. High degree of awareness about possibility of muscle hernia can help in early diagnosis which can be confirmed by dynamic sonography. ${ }^{5}$ Muscle bulge through fascia is identified and dynamic examination of muscle is possible as muscle contracts and relaxes back through fascial defect.

There is no consensus about the ideal treatment for symptomatic muscle herniations. Asymptomatic hernias require reassurance, but no specific treatment. Minimally symptomatic hernias may be treated conservatively with 
restriction of exercise and the use of an elastic support. ${ }^{8}$

Various surgical techniques have been described in literature including direct closure of the defect, wide fasciotomy, closure of the defect using autologous or synthetic grafts etc. Direct closure is a simple procedure and can be useful in small defects. However, this is often not possible in larger defects and if attempted can fail, causing a recurrence of the hernia. ${ }^{6}$ This technique is also likely to increase intra-compartmental pressure because the compartment is, in effect, made smaller. This elevation of pressure is accentuated in the postoperative period by oedema, predisposing the patient to developing compartment syndrome in the anterior compartment..$^{8-10}$

Longitudinal fasciotomy can effectively relieve the symptoms and can prevent any possibility of the

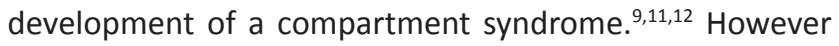
it can leave behind a cosmetically unacceptable scar and bulging. A reinforcing patch is another option. This procedure is simple, and can be used for large defects. But the use of a synthetic patch has drawbacks including cost and risk of graft intolerance to normal surrounding tissue. ${ }^{13}$ The fascial opening can also be repaired with a patch of fascia lata or periosteal graft, and can produce excellent results if good surgical techniques are employed even though donor site morbidity and compartment syndrome are remote possibilities. ${ }^{14}$

\section{CONCLUSION}

In conclusion, muscle hernias should be kept in mind as a rare differential diagnosis whenever patients present with persisting vague leg pain with or without swelling. Diagnosis is mostly clinical, but can be aided by imaging techniques like sonography. If conservative treatment fails, we recommend closure with autologous graft or fasciotomy to relieve the symptoms.

\section{REFERENCES}

1. Azar FM. Sports medicine. In: Canale ST, Beaty HJ (eds). Campbell's Operative Orthopaedic. 11(3). Philadelphia, CA: Mosby Elsevier; 2008.pg.2737-2882.

2. Schecter DC, Waddell WR, Coppinger WR. Muscle hernia: twenty personal observations. Am Surg. 1963; 29:483-490.

3. Kim M, Hong SP, Hwang SM, Park H, Ahn SK. Tibialis anterior muscle herniation developed after trauma. Int. J. Dermatol. 2008; 47: 845847.

4. Rho NK, Kim WS, Kim YJ, Yoo KH, Kim BJ, Kim MN. The use of dynamic ultrasonography for the confirmation of lower leg muscle herniation. Ann. Dermatol. 2008; 20: 190-193.

5. Agarwal A, Agarwal R. Abnormal presentation of tibialis anterior herniation. Eur. J. Orthop. Surg. Traumatol. 2006; 16: 271-272.

6. Marques A, Brenda E, Amarante TJ. Bilateral multiple muscle hernias of the leg repaired with Marlex mesh. Brit J Plast Surg. 1994; 47: 444-446.

7. Obermayer ME, Wilson JW. Fascial hernias of the legs. JAMA. 1951; 145: 548-549.

8. Wolfort GF, Mogelvang C, Filtzer HS. Anterior tibial compartment syndrome following muscle hernia repair. Arch Surg. 1973; 106: 9799.

9. Miniaci A, Rorabeck $\mathrm{CH}$. Tibialis anterior muscle hernia: a rationale for treatment. Can J Surg. 1987; 30: 79-80.

10. Almdahl SM, Due J, Samdal FA. Compartment syndrome with muscle necrosis following repair of hernia of tibialis anterior. Acta Chir Scand 1987; 153: 695.

11. Miniaci A, Rorabeck $\mathrm{CH}$. Compartment syndrome as a complication of repair of a hernia of the tibialis anterior. J Bone Joint Surg Am. 1986; 68: 1444-1445.

12. Bloem JJAM. The treatment of muscle hernias by fascial splitting. Brit J Plast Surg. 1976; 29:291-294.

13. Siliprandi L, Martini G, Chiarelli A, Mazzoleni F. Surgical repair of an anterior tibialis muscle hernia with Mersilene mesh. Plast Reconstr Surg. 1993;91:154-157.

14. Hartzell J. The use of living fascia transplant to repair a hernia of the tibialis anterior muscle. JAMA. 1936; 107: 492-493. 